EGU2020-9805

https://doi.org/10.5194/egusphere-egu2020-9805

EGU General Assembly 2020

(c) Author(s) 2021. This work is distributed under

the Creative Commons Attribution 4.0 License.

\title{
Comparison of semi-implicit and explicit particle in cell methods for the study of turbulence in space plasmas
}

\author{
Francesco Pucci ${ }^{1}$, Tulasi N. Parashar ${ }^{2}$, William H. Matthaeus ${ }^{2}$, and Giovanni Lapenta ${ }^{1}$ \\ ${ }^{1}$ Centre for Mathematical Plasma Astrophysics, Department of Mathematics, KU Leuven, Leuven, Belgium \\ ${ }^{2}$ Department of Physics and Astronomy, University of Delaware, Delaware, USA
}

The plasma that permeates the solar wind, the solar corona, the Earth's magnetosheath and several

other space environments is in a turbulent state. The effect of turbulence on the dynamics of such systems

is very relevant, considering that it is invoked to explain plasma heating, and particle acceleration and transport

in those environments.

From a mathematical point of view, turbulence is a non linear phenomenon whose study, in the kinetic

description of plasmas, requires the solution of the non linear Vlasov-Maxwell system of equations.

Due to the complexity of the problem, the solutions are nowadays found mainly by means of numerical simulations.

The most widely used method for the solution of the Vlasov-Maxwell system is the Particle In Cell (PIC) method.

PIC methods can be divided into two major classes: explicit and implicit, depending on the algorithm used

for advancing the solution in time.

In this work, we compare two different PIC methods that use an explicit and a semi-implicit algorithm, respectively.

The explicit method is implemented in the code P3D[1], while the semi-implicit method in code iPic3D[2].

Both methods are fully kinetic, namely they retain the kinetic effects for both ions and electrons.

The two codes are tested against a classical set up of plasma turbulence in a 2D cartesian geometry[3]. The system is initialized with a restricted number of modes at large scale and evolves in time without forcing. The box size is of several tens of ion inertial length. The grid size is of the order of the Debye length for the explicit scheme, to ensure numerical stability, and is varied across the electron skin depth for the semi-implicit, by performing different simulations.

Several analyses are presented: global energy conversion, magnetic and electric spectra, scale dependent kurtosis, 
temperature anisotropy for both species, proxies of dissipation such as J.E and PiD[4].

The weaknesses and strengths of the two methods in terms of description of the physical dynamics and of

computational time are presented, along with a convergence study of the semi-implicit to the explicit

method as the resolution of the former is varied.

[1] Zeiler, A., Biskamp, D., Drake, J. F., Rogers, B. N., Shay, M. A., \& Scholer, M. (2002). Journal of Geophysical Research: Space Physics, 107(A9), SMP-6.

[2] Markidis, S., Lapenta, G., \& Rizwan-uddin (2010). Mathematics and Computers in Simulation, 80(7), 1509-1519.

[3] Parashar, T. N., Matthaeus, W. H., \& Shay, M. A. (2018). The Astrophysical Journal Letters, 864(1), L21.

[4] Yang, Y., Matthaeus, W. H., Parashar, T. N., Wu, P., Wan, M., Shi, Y., et al. (2017). Physical Review E, 95(6), 061201. 